\title{
Generalized Beamforming for MIMO Systems with Limited Transmitter Information
}

\author{
K. Kiran Mukkavilli \\ Qualcomm inc., \\ San Diego, CA 92121 \\ mkkiran@rice.edu
}

\author{
A. Sabharwal and B. Aazhang \\ Rice University \\ Houston, TX 77005 \\ $\{$ ashu, aaz\}@rice.edu
}

\begin{abstract}
In this work, we investigate the outage performance of beamforming schemes in wireless systems equipped with multiple transmit and receive antennas. In particular, we analyze the outage performance of unit rank beamforming in the presence of finite rate channel feedback at the transmitter. Further, we present finite size beamformer codebook constructions which result in near-optimal outage performance for unit rank beamforming. The constructions obtained for the unit rank beamforming scheme are then extended to higher rank beamforming schemes with quantized channel information. We show that significant performance improvements as well as reduction in decoding complexity can result from a small number of feedback bits.
\end{abstract}

\section{Introduction}

There is an increasing demand for higher data rates on wireless communication links to support various kinds of evolving applications. Space time coding, using multiple antennas at the transmitter with one or more antennas at the receiver, has gained wide attention to meet the challenge of increasing the data rates. Telatar's work in [1] provided insights into fundamental limits on communication using multiple transmitter - multiple receiver links while Tarokh et al. [2] did some of the early work in designing space time codes.

In the analysis of space time code design criteria, it is assumed that the channel state information at the receiver (CSIR) is perfect while the channel state information at the transmitter (CSIT) is limited to only channel statistics (actual channel realization is unknown to the transmitter). CSIR requires training symbols for channel estimation while CSIT requires resources in the form of feedback from the receiver to the transmitter. It has been observed that significant performance gains, at much lower complexity at times, can be achieved in the presence of CSIT. Telatar [1] analyzed perfect CSIT while Narula et al. [3] analyzed optimal schemes with imperfect CSIT, with multiple transmit and a single receive antenna. Power control algorithms, which minimize probability of outage or maximize mutual information, were designed in $[4,5]$. The performance of beamforming with different kinds of structure on feedback information, like channel phases or channel amplitude, was analyzed in $[6,7]$.

In practice, the capacity of the feedback channel is limited to a few bytes and hence it is important to analyze the performance of transmission schemes with constraints on the feedback channel capacity. Fundamental limits on the outage performance of beamforming schemes with finite rate feedback for single receive antenna systems were derived in [8] and good constructions of beamformers are provided in $[8,9]$.

In this work, we analyze the outage performance of beamforming schemes with multiple receive antennas, while the multiple transmit antenna receiver is equipped with finite rate feedback channel from the receiver. In particular, we analyze two different transmission schemes for such a system. First, we present a unit rank beamforming scheme which approaches transmission along the dominant eigenvector of the channel as the number of feedback bits is increased. For this case, we present a lower bound on outage probability whose proof is based on the geometrical structure inherent in the problem. Second, we present extensions to higher rank beamforming schemes with finite feedback which mimic spatial water-filling scheme proposed by Telatar [1].

The rest of the paper is organized as follows. The system model is introduced in Section 2. In Section 3, we first analyze the performance of unit rank beamforming with quantized feedback with multiple transmit and receive antennas, and then present constructions for finite size beamformer codebooks. We study extensions of codebook constructions to higher rank beamforming schemes in Section 4 while we conclude in Section 5. 


\section{System Model}

Consider a system with $t$ transmit antennas and $r$ receive antennas. Let the $r \times n$ matrix $\mathbf{H}$ denote the channel between the transmit and receive antenna arrays. It is assumed that the channel fade statistics are quasi-static, i.e., the channel realization stays fixed for the duration of a frame denoted by $l$. Let $h_{i, j}$, the $i, j$ th element of $\mathbf{H}$, denote the channel coefficient from the $j$ th transmit antenna to the $i$ th receive antenna for each $i$ and $j$. The amplitude of $h_{i, j}$ is assumed to be Rayleigh distributed with variance 1 while the phase of $h_{i, j}$ is uniformly distributed between 0 and $2 \pi$ for each $i, j$. Further, the elements of $\mathbf{H}$ are i.i.d. Let $X$ denote the $n \times l$ code matrix transmitted from transmit array while $Y$ denotes the $r \times l$ matrix received at the receive array when $X$ is transmitted. Let $\eta$, a $r \times l$ matrix, denote the additive noise at the receiver which is assumed to be circular symmetric complex Gaussian with zero mean and variance 1 per complex dimension. With this notation, we can write the samples of the received signal $Y$ as

$$
Y=H X+\eta \text {. }
$$

We assume that the channel realization is known perfectly to the receiver. We also assume that a feedback channel exists from the receiver to the transmitter, with a capacity of $B$ bits per frame which is error-free. Further, suppose that the target rate of transmission is $R$ bits/sec/Hz for every frame and the transmitter is constrained in power to $P$. We assume short term power constraint thus precluding power control mechanism for this analysis [10].

The transmission strategy that will be used at the transmitter is fixed to be beamforming, while the performance metric is outage probability.

\section{Unit Rank Beamforming}

\subsection{Performance analysis}

We will first analyze the performance of unit rank beamforming in the presence of quantized feedback at the transmitter. Under unit rank beamforming, the transmitter uses a single direction to transmit all the available power, resulting in scalar encoding and hence, scalar decoding at the receiver. With unit rank beamforming, the transmitted codeword $X$ can be written as $X=x C, x \in \mathbb{C}, C \in \mathbb{C}^{t}$, where $x$ is the information bearing scalar while $C$ is the beamforming vector. Now, corresponding to $B$ feedback bits, we have a beamformer codebook $\mathcal{C}$ comprising of $N=2^{B}$ vectors given by $\left\{C_{1}, C_{2}, . ., C_{N}\right\}$. It can be shown [11] that the following quantizer minimizes the outage probability for unit rank beamforming.
Lemma 1 For any beamformer codebook $\mathcal{C}=$ $\left\{C_{1}, C_{2}, . ., C_{N}\right\}$, the outage probability for unit rank beamforming is minimized by choosing for each channel realization $\mathbf{H}$, the vector $C_{i} \in \mathcal{C}$ which minimizes $\left\|\left\langle H, C_{i}\right\rangle\right\|_{2}$, where $\langle u, v\rangle=u v^{\dagger}$ and $\|\cdot\|_{2}$ is the $l_{2}$ norm on $\mathbb{C}^{t}$.

Using such a quantizer, and exploiting the geometry associated with the unit rank beamforming methodology, we arrive at a lower bound on the outage probability for unit rank beamforming in the case of multiple transmit and receive antennas [11]. The outage lower bound assumes the following form for the case of $t$ transmit antennas and 2 receive antennas. The proof methodology used can be extended to any number of receive antennas.

Theorem 1 Consider a wireless communication system with $t$ transmit antennas and 2 receive antennas operating with a finite size beamformer codebook of size $N=2^{B}$, where $B$ is the number of feedback bits available for each channel realization. Assuming a quasistatic flat fading channel with Rayleigh fading statistics between each pair of transmit element and receive element, the outage probability of such a system operating at SNR $P$ and transmission rate $R$ is bounded below as

$$
\begin{aligned}
P_{\text {out }}(R, P) \geq & 1-N\left(1+\gamma_{0}\right) e^{-\gamma_{0}} \\
+ & e^{-\gamma_{1}}\left(\sum_{k=0}^{2 t-1} \frac{N\left(1+\gamma_{0}\right)\left(\gamma_{1}-\gamma_{0}\right)^{k}-\gamma_{1}^{k}}{k !}\right) \\
& -N e^{-\gamma_{1}} \gamma_{0} \frac{\left(\gamma_{1}-\gamma_{0}\right)^{2 t-1}}{(2 t-1) !}
\end{aligned}
$$

where $\gamma_{0}$ and $\gamma_{1}$ are such that

$$
\begin{gathered}
\gamma_{0}=\frac{2^{R}-1}{P} \\
N\left(1-\frac{\gamma_{0}}{\gamma_{1}}\right)^{2 t-2}\left(1+(2 t-2) \frac{\gamma_{0}}{\gamma_{1}}\right)=1 .
\end{gathered}
$$

We will now discuss the construction of beamformer codebooks whose performance approaches the performance predicted by the above theorem.

\subsection{Unit rank beamformer construction}

In this section, we heuristically argue that the beamformer codebook construction criterion for unit rank beamforming with multiple transmit and receive antennas should be similar to design criterion for good beamformer codebooks with multiple transmitter and a single receive antenna presented in [8]. Our arguments are further justified by our subspace packing 
based constructions, first proposed for a MISO system [8], which result in excellent performance even in MIMO systems with unit rank beamforming.

Given a beamformer codebook $\mathcal{C}=C_{1}, C_{2}, . . C_{N}$ and a channel realization $\mathbf{H}$, the quantization rule (from Lemma 1) picks the vector $C_{i}$ which maximizes the matrix-vector product of the channel with the elements of $\mathcal{C}$. This quantization rule bears resemblance with the well known Rayleigh quotient in matrix theory [12]. In particular, if $V_{1}$ is a column vector with unit norm, then $\left\|\mathbf{H} V_{1}\right\|_{2}$ is maximized (and equals the maximum eigenvalue of $\mathbf{H}$ ) when $V_{1}$ is given by the principal eigenvector of $\mathbf{H}^{\dagger} \mathbf{H}$. In our case, the quantization rule is essentially performing the equivalent maximization over the constrained set of the finite sized beamformer codebook. As a result, the quantization rule picks the best approximation of the principal eigenvector of $\mathbf{H}^{\dagger} \mathbf{H}$ in the beamformer codebook. Further, as $N$ (number of vectors in $\mathcal{C}$ ) gets very large, a degenerate beamformer codebook spans all the available directions equally well and the quantization rule does in fact result in the principal eigenvector.

It can be shown that if (the column vector) $V_{p}$ is the principal eigenvector of $\mathbf{H}^{\dagger} \mathbf{H}$, then

$$
\arg \max _{C_{i} \in \mathcal{C}}\left|\left\langle V_{p}^{\dagger}, C_{i}\right\rangle\right|=\arg \max _{C_{i} \in \mathcal{C}}\left\|\left\langle\mathbf{H}, C_{i}\right\rangle\right\|_{2} .
$$

Hence, for the sake of beamformer construction and the quantization rule, the channel is effectively reduced a vector channel characterized by its principal eigenvector. For vector channels, we have shown [8] that good beamformer codebooks result from the solution to the subspace packings problem. Following the above arguments, the beamformer design criterion for the MISO channel should also extend to the MIMO channel when employing unit rank beamforming. In particular the beamformer design criterion is given by the following proposition [8].

Proposition 1 (Design criterion) The design criterion for a good beamformer codebook $\mathcal{C}$ comprising of $N$ beamforming vectors for $t$ transmit antennas is given by

$$
\mathcal{C} \in \underbrace{\mathbb{C}^{t} \times \ldots \times \mathbb{C}^{t}}_{N} \max _{\substack{i, j: \\ 1 \leq i \neq j \leq N}}\left|\left\langle C_{i}, C_{j}\right\rangle\right| .
$$

The above design criterion suggests that the design of good beamformer codebooks for MIMO systems depends only on the number of transmit antennas and is independent of the number of receive antennas. However, note that the actual performance of the resulting beamformer codebooks depends both on the number of the transmit and receive antennas. For instance, the diversity gain obtained with sufficient number of beamforming vectors will be $t \times r$ which depends both on the number of receive and transmit antennas.

We have also shown that under certain conditions [8], the above design criterion is equivalent to the design criterion of unitary space time constellations for non-coherent constellations. Hence, all the constructions available for unitary constellation design can also be used for the beamformer design problem with the quantization metric given by Lemma 1 .

Figure 1 shows the performance of the quantized unit rank beamformers with four transmit antennas and two receive antennas transmitting at $R=$ $4 b i t s / s e c / H z$. The performances of space-time coding scheme which does not use any channel state information as well as the spatial water-filling solution which requires complete channels state information are also given for comparison. With 6 bits of feedback information, we see a gain of about $0.8 \mathrm{~dB}$ for the unit rank beamforming scheme over the space time coding scheme at an outage performance of $10^{-3}$. The gains increase further as the number of feedback bits is increased. The performance gains are in addition to significant reduction in complexity for the beamforming scheme over the space time coding as already explained. The performance of dominant eigenvector beamforming which is the limit of the unit rank beamforming as the number of beamforming vectors gets large is also given in Figure 1.

In the next section, we will investigate spatial water-filling schemes (essentially higher rank beamforming schemes) when finite rate feedback is available at the transmitter.

\section{Generalized Beamforming with Fi- nite Rate Feedback}

Let us suppose that the transmitter is equipped with $t$ transmit antennas while the receiver is equipped with two receive antennas. Let $\mathbf{H}$ correspond to a sample channel realization. Further, we assume the existence of a feedback channel with $B$ bits. We adopt a decoupled approach to the quantization problem where the quantizers used for the eigenvectors and the eigenvalues are independent from each other. Such a separation imposes certain structure on the quantizer design, which can reduce the complexity of implementation of the quantizer in practice. Let $V_{1}$ and $V_{2}$ correspond to the eigenvectors of $\mathbf{H}^{\dagger} \mathbf{H}$, while $\lambda_{1}$ and $\lambda_{2}$ are the corresponding eigenvalues with $\lambda_{1} \geq \lambda_{2}$. Let $P_{1}$ and $P_{2}$ denote the power levels determined by the water-filling algorithm [1] for a total power of $P$.

First of all, we argue that it is beneficial to quantize 


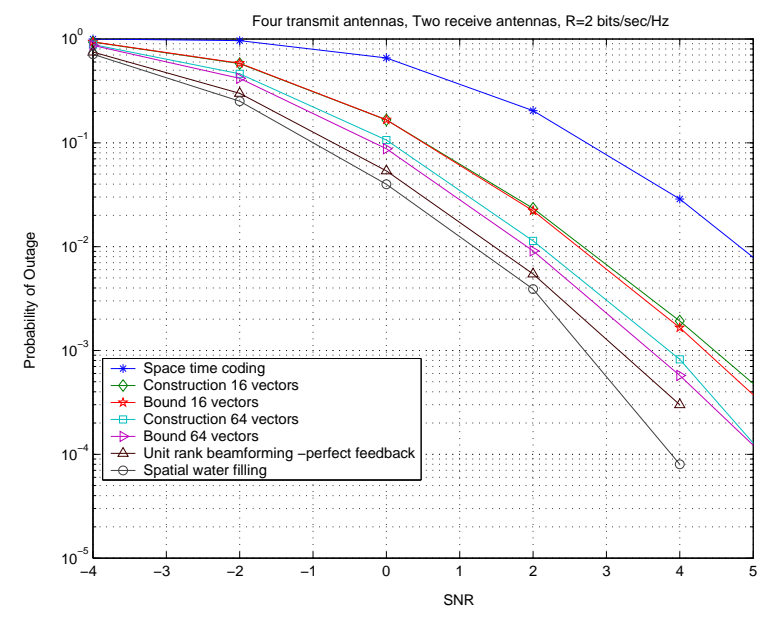

Figure 1: Comparison of performance of space time codes with various unit rank beamforming schemes for 4 transmit and 2 receive antenna systems transmitting at $\mathrm{R}=2 \mathrm{bits} / \mathrm{sec} / \mathrm{Hz}$.

the power levels $P_{1}$ and $P_{2}$ instead of the eigenvalues. Indeed, the water-filling algorithm requires the absolute values of the eigenvalues and hence partial information such as the ratio of the eigenvalues, for example, will not suffice. On the other hand, consider the situation where the water-filling algorithm is performed at the receiver and only the power levels are quantized. In this case, it suffices to quantize the ratio of the power levels $\frac{P_{2}}{P_{1}}$ since the total power available $\left(P_{1}+P_{2}=P\right)$ is known at the transmitter. Further savings in feedback bits can be obtained by noting that $P_{1} \geq P_{2}$ so that $\rho=\frac{P_{2}}{P_{1}}$ always lies between 0 and 1 .

The quantizer for the power distribution vector is given in Table 1. Note that we set $P_{2}=\rho P_{1}$, where $0 \leq \rho \leq 1$, with $P_{1} \geq 0.5 P$, where 2 bits are used to describe $\rho$. We essentially exploit the knowledge that the eigenvalue corresponding to the principal eigenvector will get at least half of the available power for transmission. We then allocate the remaining half of the transmission power based on the strength of the second eigenvalue in comparison with the first eigenvalue. We use a uniform quantizer for ratio of the eigenvalues as well as the remaining transmission power.

We will now discuss the quantization of the two active eigenvectors of $H^{\dagger} H$. Consider a finite size beamformer codebook $\mathcal{C}$ of size $N$ constructed as discussed in the previous section. We can first apply the quantization rule discussed in the last section to determine the best approximation to the principal eigenvector among the available vectors in $\mathcal{C}$. Note that the

\begin{tabular}{|c|c|c|}
\hline Eigenvalue ratio & $\left(P_{1}, P_{2}\right)$ & $\rho$ \\
\hline $0.75 \leq \frac{\lambda_{2}}{\lambda_{1}} \leq 1$ & $\left(\frac{1}{2}, \frac{1}{2}\right)$ & 1 \\
$0.5 \leq \frac{\lambda_{2}}{\lambda_{1}} \leq 0.75$ & $\left(\frac{4}{6}, \frac{2}{6}\right)$ & $\frac{1}{2}$ \\
$0.25 \leq \frac{\lambda_{2}}{\lambda_{1}} \leq 0.5$ & $\left(\frac{5}{6}, \frac{1}{6}\right)$ & $\frac{1}{5}$ \\
$0 \leq \frac{\lambda_{2}}{\lambda_{1}} \leq 0.25$ & $(1,0)$ & 0 \\
\hline
\end{tabular}

Table 1: Quantizer used for the power allocation vector in the case of 4 transmit antennas and 2 receive antennas.

specification of this vector at the transmitter requires $\log _{2}(N)$ feedback bits. However, we can gain substantially in the specification of the second eigenvector by noting the following useful property.

Note that the eigenvectors of $H^{\dagger} H$ lie in $\mathbb{C}^{t}$. Further, the eigenvectors are all mutually orthogonal. Hence, the specification of the first eigenvector determines the subspace which contains the second active eigenvector. In particular, the second eigenvector lies in the $t-1$ dimensional subspace which is orthogonal to the principal eigenvector. Hence, we can improve the description of the second vector significantly by constructing a second codebook in $t-1$ dimensions instead of the original $t$ dimensional space.

However, it is not desirable to modify the composition of the codebook of the second eigenvector based on the first eigenvector, since the orthogonal subspace of the second vector depends on the principal eigenvector. We present an algorithm where both the beamformer codebooks are independent of the actual channel realization.

\subsection{Algorithm for eigenvector quantiza- tion}

Let $\mathcal{C}_{1}$ be a beamformer codebook in $\mathbb{C}^{t}$ comprising of $N_{1}=2^{B_{1}}$ vectors. Similarly, let $\mathcal{C}_{2}$ be a beamformer codebook in $\mathbb{C}^{t-1}$ comprising of $N_{2}=2^{B_{2}}$ vectors. Let $\mathbf{H}$ be the channel realization, while $V_{1}$ and $V_{2}$ are the active eigenvectors of $\mathbf{H}^{\dagger} \mathbf{H}$.

We first quantize $V_{1}$ in $\mathcal{C}_{1}$ using the quantization rule given by Lemma 1 . In particular, we pick $C_{i}^{1} \in \mathcal{C}_{1}$ (note that the superscript corresponds to the codebook index) such that $\left\|\left\langle\mathbf{H}, C_{i}^{1}\right\rangle\right\|_{2}$ is the maximum for all the vectors in $\mathcal{C}_{1}$. Without loss of generality, we assume that $C_{1}^{1}$ maximizes the inner product with $\mathbf{H}$ among all the vectors in $\mathcal{C}_{1}$.

Now, consider the vectors in $\mathcal{C}_{2}$. We construct a codebook $\mathcal{C}_{2}^{\prime}$ from $\mathcal{C}_{2}$ such that $\mathcal{C}_{2}^{\prime}$ lies in $\mathbb{C}^{t}$. Hence, $\mathcal{C}_{2}$ is an embedding of $\mathcal{C}_{2}^{\prime}$ in $\mathbb{C}^{t-1}$. By construction, $\mathcal{C}_{2}^{\prime}$ is such that the first co-ordinate of all the vectors is set to zero. Hence, the vectors in $\mathcal{C}_{2}^{\prime}$ lie in the or- 
thogonal subspace of the axis $[1,0, . ., 0]$ of $\mathbb{C}^{t}$. Further, the embedding rule of $\mathcal{C}_{2}^{\prime}$ into $\mathcal{C}_{2}$ is that the first coordinate of $\mathcal{C}_{2}^{\prime}$ is dropped to obtain the corresponding vector in $\mathcal{C}_{2}$. Hence, if $C_{i}^{2^{\prime}}=\left[0, c_{1}, c_{2}, . . c_{t-1}\right]$, then the corresponding $C_{i}^{2}$ in $\mathcal{C}_{2}$ is given by $\left[c_{1}, c_{2}, . . c_{t-1}\right]$.

Now, we make use of the property that $\mathcal{C}_{2}^{\prime}$ is in the orthogonal subspace of $e_{1}=[1,0, \ldots 0]$ in $\mathbb{C}^{t}$. In particular, we rotate the vectors in $\mathcal{C}_{1}$ such that $C_{1}^{1}$ coincides with $e_{1}$. Let $A$ be a $t \times t$ unitary matrix, constructed in a predetermined fashion from $C_{1}^{1}$ such that $A^{\dagger} C_{1}^{1}=e_{1}$. Now, we rotate the channel matrix $\mathbf{H}$ by the same matrix $A$ before we quantize the second vector. Equivalently, we rotate the second vector $V_{2}$ by the matrix $A$ to give $V_{2}^{\prime}=A^{\dagger} V_{2}$. Now, we quantize $V_{2}^{\prime}$ in the second beamformer codebook $\mathcal{C}_{2}^{2^{\prime}}$. Suppose $C_{k}^{2^{\prime}}$ is the vector in $\mathcal{C}_{2}^{2^{\prime}}$ which maximizes the inner product with $V_{2}^{\prime}$. Then, the transmitter gets the label $k$ and the transmitter uses $A\left(C_{2}^{\prime}\right)^{T}$ for transmission, where the superscript $T$ stands for matrix transpose operation. Note that $A$ is a function of $C_{1}^{1}$ only and since the transmitter has information about $C_{1}^{1}$ via feedback channel, the matrix $A$ can be reproduced at the transmitter. Hence, both the resulting codebooks, $\mathcal{C}_{1}$ and $\mathcal{C}_{2}$ are independent of the actual channel realization.

The performance of the a rank two beamformer using 2 and 3 bit quantizers for the eigenvectors, is shown in Figure 2. Note that the performance gains can be improved further with higher number of feedback bits.

\section{Conclusion}

In this paper, we have presented performance analysis as well as constructions of finite size beamformer codebooks with multiple transmit and receive antenna systems. Starting with more regular and structured case of unit rank beamforming schemes, we extended our constructions to the more general case of higher rank beamforming schemes which are known to be optimal for MIMO systems [1].

\section{References}

[1] E. Telatar, "Capacity of multi-antenna gaussian channels," ATET-Bell Labs Internal Tech. Memo, June 1995.

[2] V. Tarokh, N. Seshadri, and A. Calderbank, "Spacetime codes for high data rate wireless communication:performance criterion and code construction," IEEE Transactions on Information Theory, vol. 44, pp. 744-765, March 1998.

[3] A. Narula, M.D. Trott, and G.W. Wornell, "Performance limits of coded diversity methods for transmitter antenna arrays," IEEE Transactions on Information Theory, pp. 2418-2433, November 1999.

[4] S. Bhashyam, A. Sabharwal, and B. Aazhang, "Feedback gain in multiple antenna systems," IEEE Transactions on communications, vol. 50, pp. 785-798, May 2002.

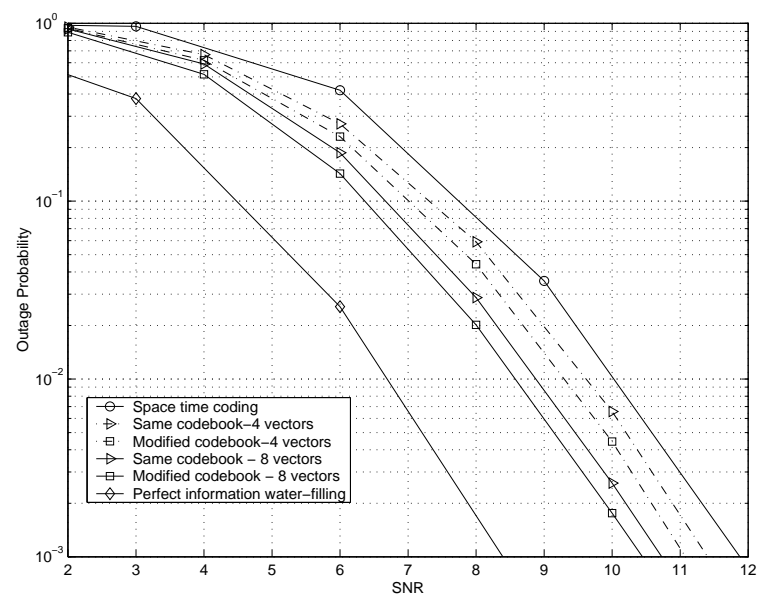

Figure 2: Rank two beamforming for 4 transmit antennas and 2 receive antennas.

[5] A. Sabharwal, E. Erkip, and B. Aazhang, "On channel state information in multiple antenna block fading channels," in ISITA, Hawaii, 2000.

[6] K. K. Mukkavilli, A. Sabharwal, and B. Aazhang, "Design of multiple antenna coding schemes with channel feedback," in Thirty fifth Asilomar Conference on Signals, Systems and Computers, 2001, pp. 1009-1013.

[7] R.W. Heath Jr. and A. Paulraj, "A simple scheme for transmit diversity using partial channel feedback," in Thirty Second Asilomar Conference on Signals, Systems and Computers, 1998, pp. 1073-1078.

[8] K. K. Mukkavilli, A. Sabharwal, E. Erkip and B.Aazhang, "On Beamforming with Finite Rate Feedback in Multiple Antenna Systems," IEEE transactions on Information Theory, vol. 49, no. 10, pp. 2562-2579, October 2003.

[9] D. J. Love, R. W. Heath, and T. Strohmer, "Grassmannian beamforming for multiple-input multiple-output wireless systems," IEEE Transactions on Information Theory, vol. 49 , no. 10 , pp. 2735-2746, October 2003.

[10] G. Caire, G. Taricco, and E. Biglieri, "Optimum power control over fading channels," IEEE Transactions on Information Theory, vol. 45, no. 5, pp. 1468-1489, July 1999.

[11] K. K. Mukkavilli, A. Sabharwal and B.Aazhang, "Design and Performance Analysis of Beamforming with MIMO Systems," Under preparation.

[12] G. H. Golub and C. F. Van Loan, Matrix Computations, The Johns Hopkins University Press, Baltimore, 1983. 